\title{
BMJ Open Short-term abstinence from alcohol and changes in cardiovascular risk factors, liver function tests and cancer-related growth factors: a prospective observational study
}

Gautam Mehta, ${ }^{1}$ Stewart Macdonald, ${ }^{1}$ Alexandra Cronberg, ${ }^{2}$ Matteo Rosselli, ${ }^{1}$ Tanya Khera-Butler, ${ }^{2}$ Colin Sumpter, ${ }^{2}$ Safa Al-Khatib, ${ }^{1}$ Anjly Jain, ${ }^{3}$ James Maurice, ${ }^{1}$ Christos Charalambous, ${ }^{1}$ Amir Gander, ${ }^{4}$ Cynthia Ju, ${ }^{5}$ Talay Hakan, ${ }^{6}$ Roy Sherwood, ${ }^{7}$ Devaki Nair, ${ }^{8}$ Rajiv Jalan, ${ }^{1}$ Kevin P Moore ${ }^{1}$

To cite: Mehta G, Macdonald S, Cronberg A, et al. Short-term abstinence from alcohol and changes in cardiovascular risk factors, liver function tests and cancer-related growth factors: a prospective observational study. BMJ Open 2018;8:e020673. doi:10.1136/ bmjopen-2017-020673

- Prepublication history and additional material for this paper are available online. To view these files, please visit the journal online (http://dx.doi. org/10.1136/bmjopen-2017020673).

Received 16 November 2017 Revised 2 March 2018 Accepted 5 April 2018
Check for updates

For numbered affiliations see end of article.

Correspondence to

Dr Gautam Mehta;

gautam.mehta@ucl.ac.uk

\section{ABSTRACT}

Objective To assess changes in metabolic risk factors and cancer-related growth factors associated with short-term abstinence from alcohol.

Design Prospective, observational study.

Setting Single tertiary centre.

Participants Healthy subjects were recruited based on intention to: (1) abstain from alcohol for 1 month (abstinence group), or (2) continue to drink alcohol (control group). Inclusion criteria were baseline alcohol consumption $>64 \mathrm{~g} /$ week (men) or $>48 \mathrm{~g} /$ week (women). Exclusion criteria were known liver disease or alcohol dependence.

Primary and secondary outcome measures The primary outcome was change in insulin resistance (homeostatic model assessment (HOMA) score). Secondary outcomes were changes in weight, blood pressure (BP), vascular endothelial growth factor (VEGF), epidermal growth factor (EGF) and liver function tests. Primary and secondary outcomes were adjusted for changes in diet, exercise and cigarette smoking.

Results The abstinence group comprised 94 participants (mean age 45.5 years, $\mathrm{SD} \pm 1.2$ ) and the control group 47 participants (mean age 48.7 years, $S D \pm 1.8$ ). Baseline alcohol consumption in the abstinence group was $258.2 \mathrm{~g} /$ week, $\mathrm{SD} \pm 9.4$, and in the control group $233.8 \mathrm{~g}$, $\mathrm{SD} \pm 19$.0. Significant reductions from baseline in the abstinence group (all $p<0.001$ ) were found in: HOMA score (-25.9\%, IQR $-48.6 \%$ to $+0.3 \%)$, systolic BP $(-6.6 \%$, IQR $-11.8 \%$ to $0.0 \%)$, diastolic BP $(-6.3 \%$, IQR $-14.1 \%$ to $+1.3 \%)$, weight $(-1.5 \%$, IQR $-2.9 \%$ to $-0.4 \%)$, VEGF $(-41.8 \%$, IQR $-64.9 \%$ to $-17.9 \%)$ and EGF $(-73.9 \%$, IQR $-86.1 \%$ to $-36.4 \%$ ). None of these changes were associated with changes in diet, exercise or cigarette smoking. No significant changes from baseline in primary or secondary outcomes were noted in the control group. Conclusion These findings demonstrate that abstinence from alcohol in moderate-heavy drinkers improves insulin resistance, weight, BP and cancer-related growth factors. These data support an independent association of alcohol consumption with cancer risk, and suggest an increased
Strengths and limitations of this study

- Prospective study design.

- Recruitment of a control group.

- Thorough characterisation of the biological and lifestyle confounders.

- Lack of randomisation to groups.

- Study cohort all from university teaching hospital or science magazine.

risk of metabolic diseases such as type 2 diabetes and fatty liver disease.

\section{INTRODUCTION}

Alcohol is a major cause of disability and preventable death. Globally, alcohol is the seventh leading risk factor overall in terms of disability-adjusted life years (DALYs), and is the leading risk factor globally in working age individuals (ages 15-59). Moreover, alcohol use-attributable DALYs have increased by over $25 \%$ in the last 25 years. ${ }^{1}$ European countries have among the highest alcohol consumption. Eastern Europe has the highest per capita consumption worldwide, ${ }^{2}$ and in the UK over $25 \%$ of the adult population drink in excess of recommended guidelines. ${ }^{3}$

Aside from liver disease, which is the third most common cause of preventable death in the UK, there is also a significant burden from alcohol-related cancer and metabolic syndrome. ${ }^{3}$ Alcohol has been classified by the WHO as a class I carcinogen for some decades, and a report from the World Cancer Research Fund/American Institute for Cancer Research states that there is convincing evidence that alcohol is causally 
related to cancers of the oral cavity, pharynx, larynx, oesophagus, breast and colorectum. ${ }^{4}$

Moreover, it has long been recognised that there is an important interaction between alcohol misuse and fatty liver disease. ${ }^{5}$ One of the main factors driving the development of fatty liver disease and steatohepatitis is insulin resistance. Thus, any action that improves insulin resistance will have a major impact on the development and severity of fatty liver disease. However, there remains debate as to the impact of alcohol consumption on fatty liver disease driven predominantly by insulin resistance and metabolic factors. ${ }^{67}$

In this climate of increased awareness of alcohol-related morbidity, the UK Chief Medical Officers have revised downwards their weekly guidance limits. ${ }^{8}$ Additionally, public health campaigns, where non-dependent drinkers are encouraged to commit to short-term abstinence from alcohol, are increasingly common. The aim of this study was to assess changes in insulin resistance, metabolic risk factors and cancer-related growth factors with short-term abstinence from alcohol in moderate drinkers.

\section{METHODS}

\section{Study design}

This was a single-centre, prospective, observational study conducted at the Royal Free London NHS Foundation Trust. Written informed consent was obtained from all participants. Study recruitment was initiated through email advertising within University College London, Queen Mary University of London and New Scientist Magazine. The entry criteria were baseline alcohol consumption of $>64 \mathrm{~g} /$ week (eight units) for men or $>48 \mathrm{~g} /$ week (six units) for women. Exclusion criteria were $>3$ days abstinence from alcohol prior to commencement of the study, the presence of known liver disease or alcohol dependence. Participants were not randomised to group, but were allocated based on intention to maintain abstinence for 1 month (abstinence group) or to continue alcohol consumption (control group).

Participants were assessed at baseline, and after 1 month. The primary outcome was change in insulin resistance (homeostatic model assessment (HOMA) score) at baseline and 1 month. Secondary outcomes were changes in weight, blood pressure (BP), vascular endothelial growth factor (VEGF), epidermal growth factor (EGF) and liver function tests. Information on diet, exercise and smoking history were obtained by self-reporting using components of the Simple Lifestyle Indicator Questionnaire (SLIQ). ${ }^{9}$ Self-reported alcohol intake was assessed at baseline using the full Alcohol Use Disorders Identification Test (AUDIT) questionnaire, and a direct interview by a single interviewer $(\mathrm{KM})$ was also conducted to assess alcohol intake over the preceding 2 months, using the timeline follow back method. ${ }^{10}$ Additionally, a follow-up telephone interview was conducted at 6-8 months to determine drinking habits following the study period, using the full
AUDIT questionnaire (modified to capture data for the preceding 6-8 months).

Sample size calculation for the control group was performed, based on pre/post data acquired from the abstinence group (table 1). Specifically, based on these data, a power calculation determined that the following sample sizes were required to detect statistically significant differences of the same magnitude $(80 \%$ power, alpha 5\%, two-sided test): HOMA score $\mathrm{n}=47$, weight $\mathrm{n}=21$, VEGF $\mathrm{n}=31$, EGF $\mathrm{n}=30$.

$\mathrm{BP}$ was measured seated, following a $2 \mathrm{~min}$ rest period, and the mean of three measurements was recorded. Fasting blood was taken, between 08:00 and midday, for measurement of glucose, insulin, liver function tests, lipids, carbohydrate deficient transferrin (abstinence group only) and VEGF (isoforms 165, 145 and 121) and EGF (Randox Investigator, Randox, Belfast, UK). The HOMA score was calculated according to the methods of Matthews et al. ${ }^{11}$ Participants with diabetes requiring treatment were excluded from HOMA measurements.

\section{Statistical analysis}

Baseline and 1-month differences were analysed by paired t-test for normally distributed differences in continuous variables, by Wilcoxon signed-rank test for non-normally distributed differences in continuous variables, and differences in categorical variables by $\mathrm{X}^{2}$ test. Differences between abstinence and control groups were analysed by unpaired t-test for normally distributed variables, and Mann-Whitney test for variables that were not normally distributed. Lifestyle factors were categorically graded (better/same/worse), and delta change in biological variables between lifestyle groups was assessed by Kruskal-Wallis test. Multivariable logistic regression analysis was also used to test the effect of abstinence on improvement in HOMA, weight, BP, VEGF and EGF once other lifestyle factors (diet and exercise) were taken into account. Correlation between biological variables was assessed by Spearman's correlation. All analyses were performed using STATA V.13.1 and SPSS Statistics V.21.0. $\mathrm{SD}$ is reported for means and IQR for medians where applicable. All $p$ values are two sided; $p<0.01$ was considered significant to account for multiple comparisons.

\section{Patient and public involvement}

The research question was developed following public feedback to a pilot project, conducted in collaboration with, and published by, New Scientist magazine (New Scientist, 31 December 2013). Additionally, the research question was informed by focus groups, funded through the National Institute for Health Research Enabling Involvement Fund. No specific patient advisers were involved in the design or conduct of the study. Results of the study will be disseminated to all participants by email.

\section{RESULTS}

Ninety-seven participants were recruited to the abstinence group, and forty-eight participants to the control 


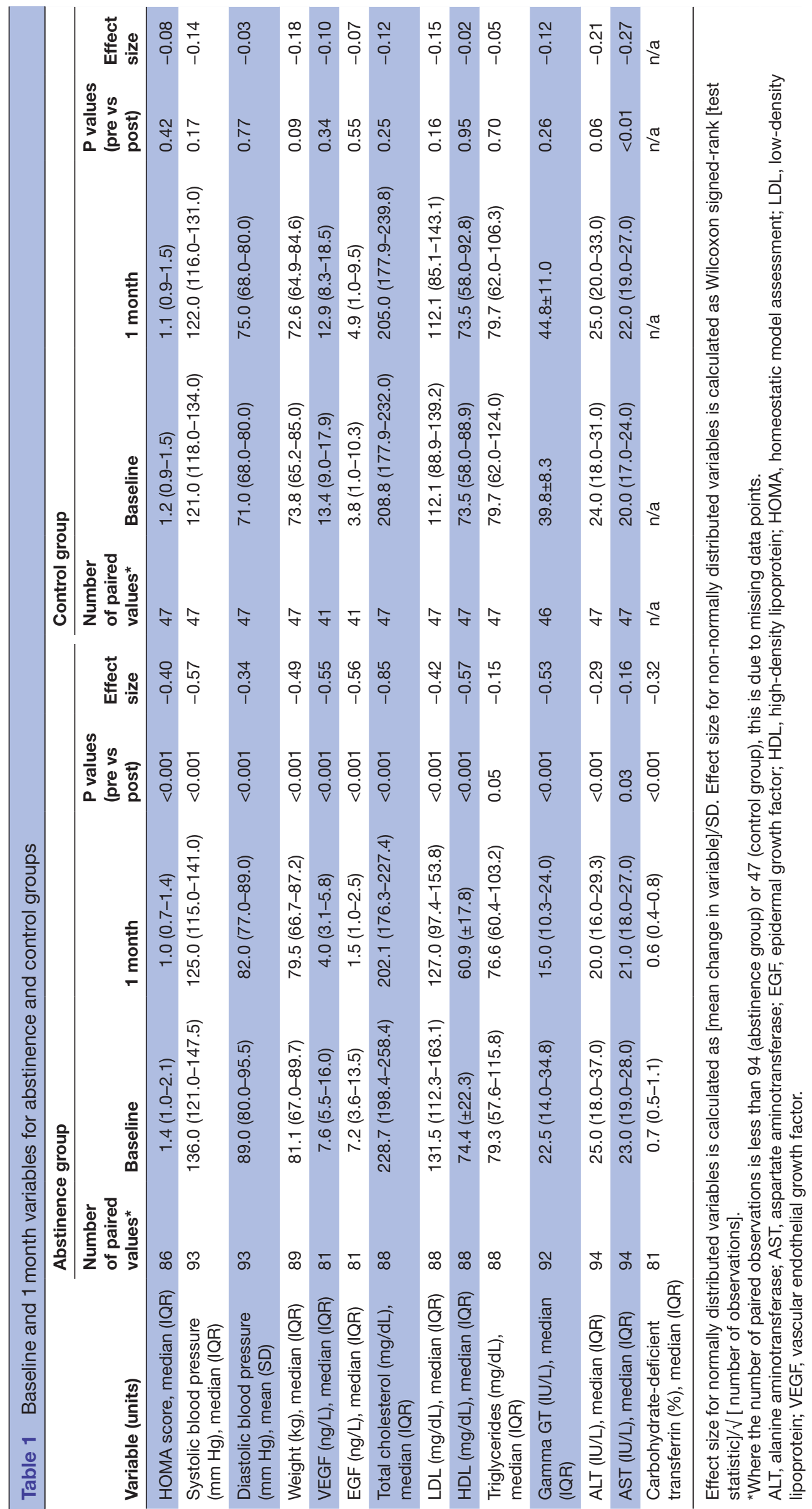




\section{Abstinence Group}

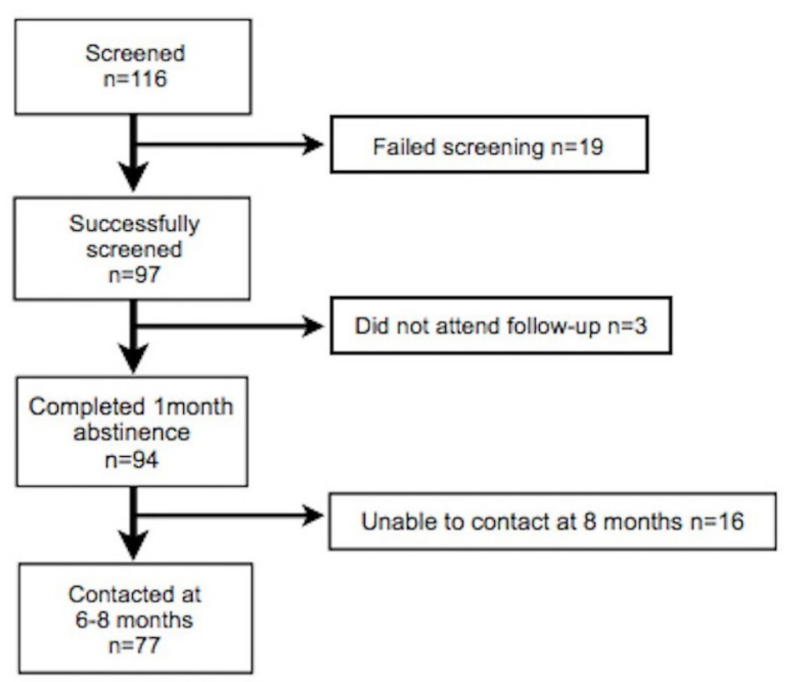

Figure 1 Flow chart of study participants.

group. Three subjects in the abstinence group and one subject in the control group did not attend for follow-up. Thus, the final abstinence group comprised 94 participants (43 men, 51 women) mean age 45.5 years, $\mathrm{SD} \pm 1.2$, and the control group comprised 47 participants (22 men, 25 women) mean age 48.7 years, $\mathrm{SD} \pm 1.8$. Mean baseline alcohol intake for the abstinence group was $258.2 \mathrm{~g}$ /week, SD \pm 9.4 (men 275.9, SD \pm 25.5 ; women 243.1, SD \pm 12.8 ). All subjects in this group, except one individual, remained abstinent for the study period-this participant was included in all analyses. Mean weekly baseline alcohol intake for the control group was $233.8 \mathrm{~g}$, $\mathrm{SD} \pm 19.0$ (men 270.2, $\mathrm{SD} \pm 26.6$; women $200.2, \mathrm{SD} \pm 25.8$ ), and was not significantly different at 1 month $260.1 \mathrm{~g}$, $\mathrm{SD} \pm 20.8$ (men 286.4, $\mathrm{SD} \pm 26.6$; women $235.8, \mathrm{SD} \pm 31.1$ ), $\mathrm{p}=0.11$. A flowchart of participants and observations is shown in figure 1.

Baseline and 1-month variables for the abstinence and control groups are listed in table 1 . There were no significant differences in baseline characteristics between abstinence and control groups, aside from baseline BP which was significantly lower in the control group (systolic BP: $135.8 \mathrm{SD} \pm 1.9 \mathrm{~mm} \mathrm{Hg}$ vs $125.7 \mathrm{SD} \pm 2.0 \mathrm{~mm} \mathrm{Hg}, \mathrm{p}<0.01$; diastolic BP: $87.7 \mathrm{SD} \pm 1.2 \mathrm{~mm} \mathrm{Hg}$ vs $74.3 .7 \mathrm{SD} \pm 1.5 \mathrm{~mm}$ $\mathrm{Hg}, \mathrm{p}<0.01)$. Antihypertensives were used in one participant in the abstinence group, and one participant in the control group. Lipid-lowering agents were used in two participants in the abstinence group, one subject in the control group. These participants were excluded from analyses for BP and lipids, respectively. Significant reductions from baseline (pre vs post) in the abstinence group were observed in: HOMA score $(-25.9 \%$, IQR -48.6 to $+0.3 \%)$, systolic BP $(-6.6 \%$, IQR $-11.8 \%$ to $0.0 \%)$, diastolic BP $(-6.3 \%$, IQR $-14.1 \%$ to $+1.3 \%)$ and weight $(-1.5 \%$, IQR $-2.9 \%$ to $-0.4 \%)$. HOMA score was not performed due to type 1 diabetes in one participant in the abstinence group. By chance, no participants had

\section{Control Group}

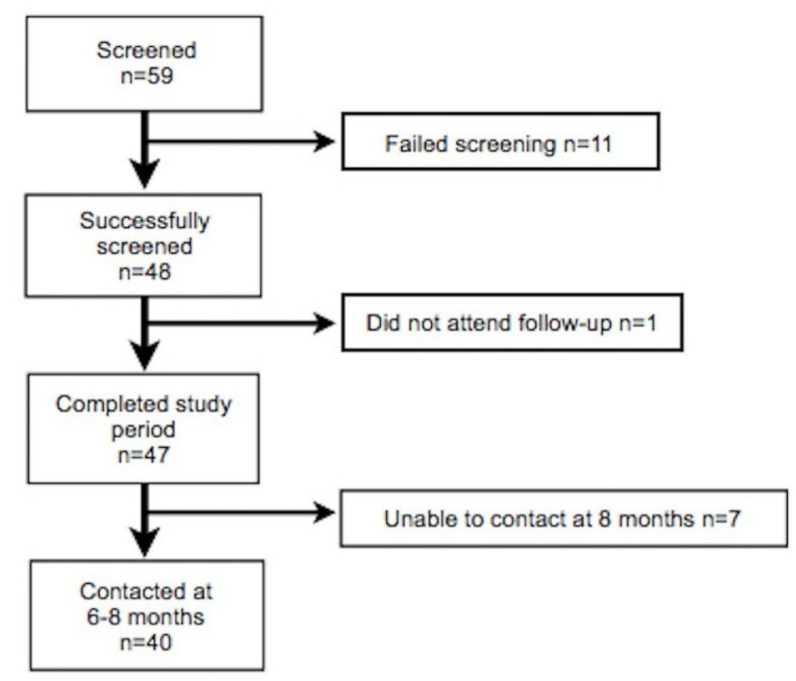

type 2 diabetes. Levels of VEGF and EGF also markedly reduced in the abstinence group, at $-41.8 \%$ (IQR $-64.9 \%$ to $-17.9 \%$ ) and $-73.9 \%$ (IQR $-86.1 \%$ to $-36.4 \%$ ) respectively (figure 2). Serum lipids (pre vs post) also improved in the abstinence group: fasting total serum cholesterol (-13.4\%, IQR $-18.9 \%$ to $-2.7 \%)$, low-density lipoprotein (LDL) cholesterol $(-9.4 \%$, IQR $-20.1 \%$ to $+4.8 \%)$, high-density lipoprotein (HDL) cholesterol $(-16.7 \%$, IQR $-25.0 \%$ to $0.0 \%$ ). All the above variables were significantly reduced from baseline, $\mathrm{p}<0.001$. By contrast, the control group did not show significant changes from baseline in any of the above variables. Changes from baseline in HOMA score, VEGF, EGF, weight and systolic and diastolic BP are shown in figure 3.

Liver function tests also improved in the abstinence group; thus, there was a significant reduction in serum alanine aminotransferase (ALT) $(-14.5 \%$, IQR -28.9 to $+6.7 \%, \mathrm{p}<0.001)$ and gamma GT $(-28.6 \%$, IQR -43.5 to $-14.4 \%, \mathrm{p}<0.001)$, and a trend towards reduction in serum aspartate aminotransferase (AST) $(-5.4 \%$, IQR -16 .two to $+9.5 \%, \mathrm{p}=0.03)$. No significant change in these variables was seen in the control group, aside from a small rise in AST $(+4.5 \%$, IQR -5.6 to $+23.1 \%$, $\mathrm{p}<0.01)$.

Lifestyle factors did not account for changes in the abstinence group. No changes were seen in exercise score $(10.9 \mathrm{SD} \pm 4.7$ vs $10.7 \mathrm{SD} \pm 4.6, \mathrm{p}=0.82)$ or cigarette smoking ( $1.3 \mathrm{SD} \pm 0.7$ vs $1.4 \mathrm{SD} \pm 0.7, \mathrm{p}=0.17$ ). A small change in diet score was noted (from 8.2 $\mathrm{SD} \pm 3.3$ to 8.8 $\mathrm{SD} \pm 3.0, \mathrm{p}=0.03$ ). The pre/post differences in HOMA score, weight, VEGF, EGF, triglycerides and HDL were distributed with a left (negative) skew. Therefore, non-parametric approaches were adopted to account for lifestyle variables. Changes in HOMA score, BP and weight in the abstinence group were not associated with changes in any lifestyle score (see online supplementary table 1 ). There was also no association between 

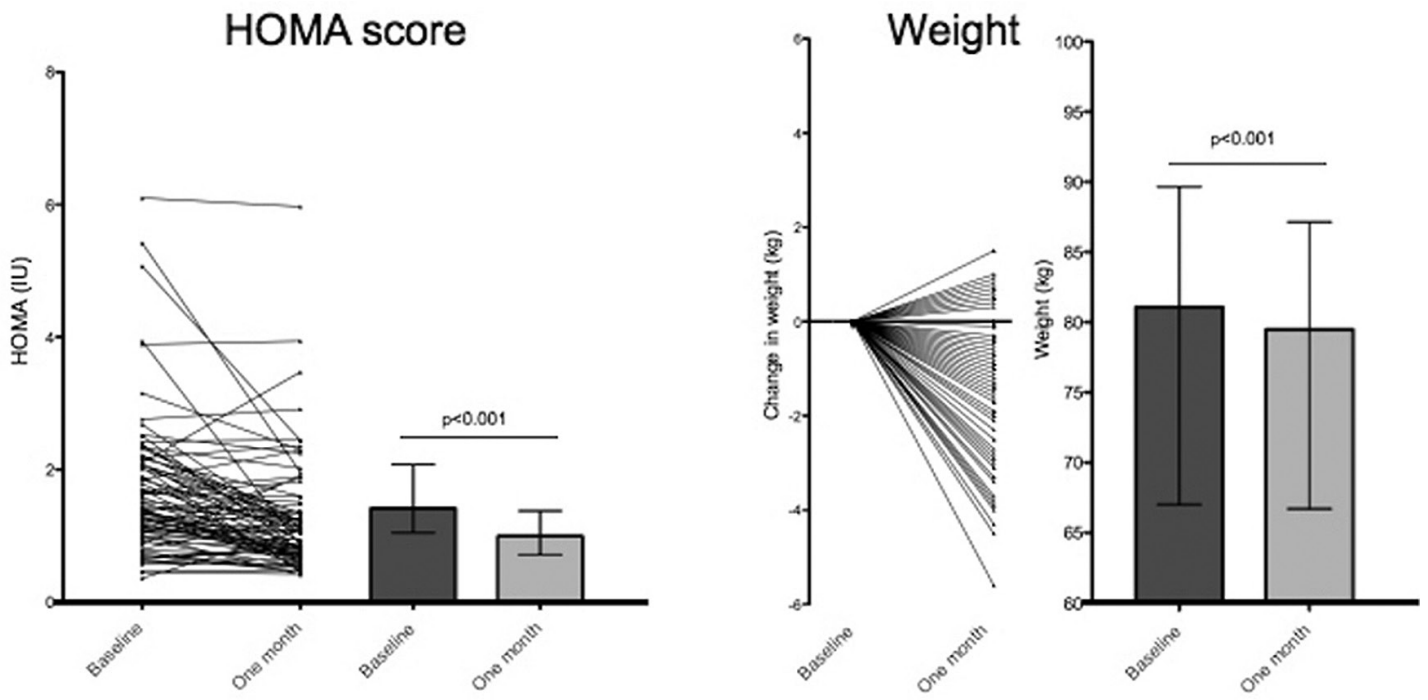

Systolic bp

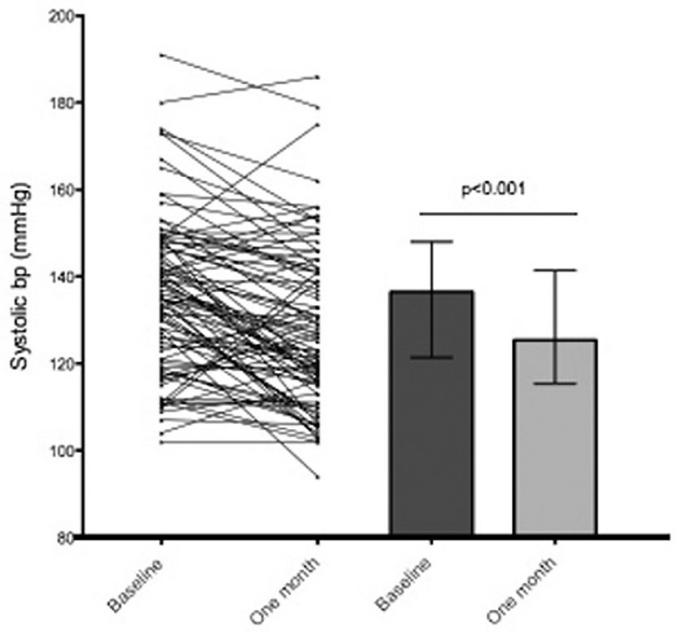

Diastolic bp
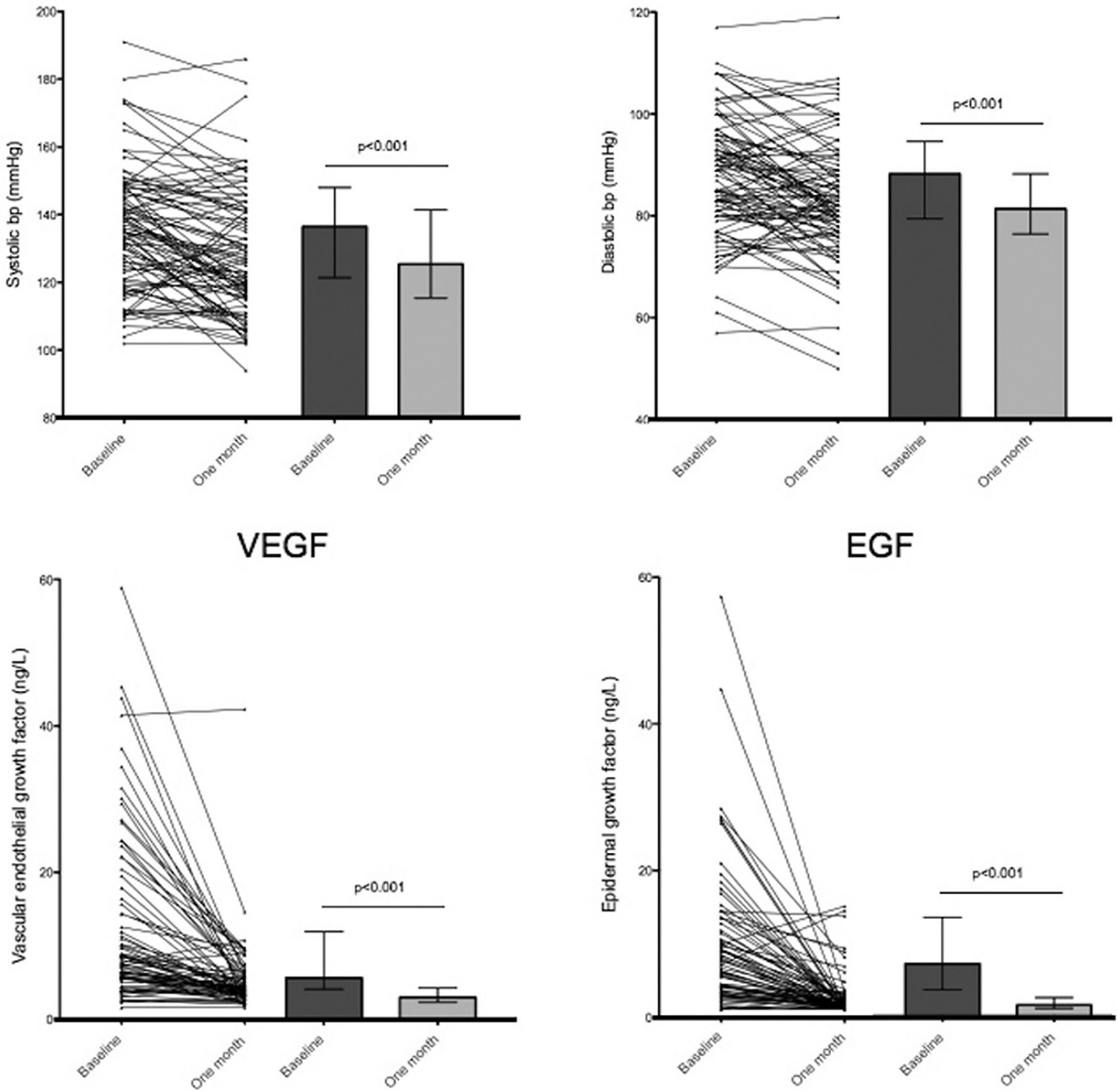

Figure 2 Baseline and 1-month data for the abstinence group presented as pre/post scatter plot (left) and bar chart chart (right). Bar chart data are presented as median (IQR). Panels (clockwise from top right): homeostatic model assessment (HOMA) score, weight, diastolic blood pressure (bp), epidermal growth factor (EGF), vascular endothelial growth factor (VEGF), systolic $\mathrm{bp}$. Baseline and 1-month values were compared with Wilcoxon signed-rank test, $p<0.01$ taken as level of significance. 

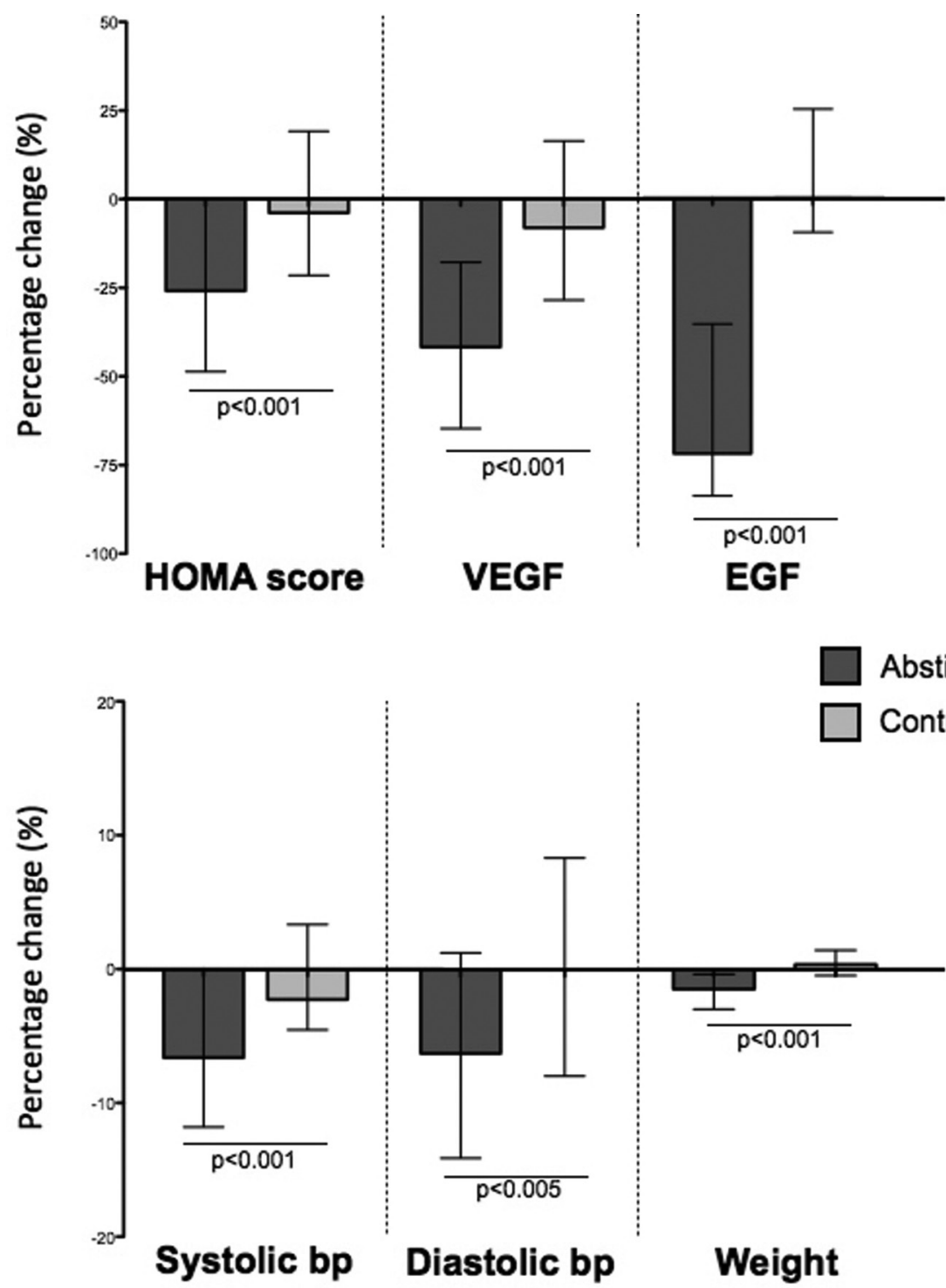

Figure 3 Percentage change from baseline in homeostatic model assessment (HOMA) score, vascular endothelial growth factor (VEGF), epidermal growth factor (EGF), systolic blood pressure (bp), diastolic bp and weight in abstinence (dark bar) and control (light bar) groups. Data are presented as median (IQR). Changes from baseline in abstinence and control groups were compared with Mann-Whitney test, $p<0.01$ taken as level of significance.

changes in HOMA score and weight $(\mathrm{r}=0.04, \mathrm{p}=0.73)$. However, changes in total cholesterol and LDL cholesterol attained borderline significance between groups when compared by change in diet in the abstinence group (see online supplementary table $1 ; \mathrm{p}=0.01$ and $\mathrm{p}=0.02$, respectively).

Additionally, multivariable logistic regression analysis was used across the whole cohort, combining the abstinence and control groups, to determine predictors of: HOMA score reduction $\geq 20 \%$, systolic BP reduction $\geq 5 \%$, weight reduction $\geq 2 \%$, VEGF reduction $\geq 20 \%$, EGF reduction $\geq 20 \%$. The model used covariates of abstinence (yes/no) or change in exercise and diet SLIQ score (better/same/worse). Abstinence was a highly significant predictor of improvement in these biological variables $($ all $\mathrm{p}<0.01)$. By contrast, change in exercise and diet score was not associated with improvement in any of these variables (table 2).

A further important result relates to follow-up questionnaire data, obtained in 77 individuals $(81.9 \%)$ in the abstinence group and $40(83.3 \%)$ in the control group, at $6-8$ months following the study period. In the abstinence group, a significant reduction in alcohol consumption was maintained from their prestudy assessment. Thus, there was a significant reduction in overall AUDIT score from 10.0 (IQR 7.0 to 15.0) to 7.0 (IQR 5.0 to 9.0), p<0.001, and in the proportion of individuals with harmful use of alcohol (AUDIT score $>8$ ) $(61.0 \%$ vs $28.5 \%, \mathrm{p}<0.001)$ at $6-8$ months compared with baseline. By contrast, in the control group, there was a non-significant trend to reduction in overall AUDIT score from 8.5 (IQR 6.3 to 12.0) to 8.0 (IQR 
Table 2 Independent predictors of improvement in HOMA
score, systolic BP, weight, VEGF and EGF

\begin{tabular}{|c|c|c|c|}
\hline \multirow{2}{*}{$\begin{array}{l}\text { Biological variable (target } \\
\text { reduction) } \\
\text { Covariate }\end{array}$} & \multicolumn{3}{|c|}{ All study participants $(n=141)$} \\
\hline & OR & $95 \% \mathbf{C l}$ & $P$ values \\
\hline \multicolumn{4}{|l|}{ HOMA score (reduction $\geq 20 \%$ ) } \\
\hline Abstinence & 3.48 & 1.60 to 7.53 & 0.002 \\
\hline Exercise+diet SLIQ score & 0.87 & 0.43 to 1.77 & 0.694 \\
\hline \multicolumn{4}{|l|}{ Systolic BP (reduction $\geq 5 \%$ ) } \\
\hline Abstinence & 6.47 & 2.71 to 15.46 & $<0.001$ \\
\hline Exercise+diet SLIQ score & 1.47 & 0.70 to 3.08 & 0.310 \\
\hline \multicolumn{4}{|l|}{ Weight (reduction $\geq 2 \%$ ) } \\
\hline Abstinence & 15.63 & 3.54 to 68.95 & $<0.001$ \\
\hline Exercise+diet SLIQ score & 0.74 & 0.323 to 1.69 & 0.475 \\
\hline \multicolumn{4}{|l|}{ VEGF (reduction $\geq 20 \%$ ) } \\
\hline Abstinence & 4.35 & 1.93 to 9.81 & $<0.001$ \\
\hline Exercise+diet SLIQ score & 2.17 & 0.97 to 4.86 & 0.59 \\
\hline \multicolumn{4}{|l|}{ EGF (reduction $\geq 20 \%$ ) } \\
\hline Abstinence & 48.81 & 15.26 to 156.06 & $<0.001$ \\
\hline Exercise+diet SLIQ score & 2.52 & 0.80 to 7.95 & 0.115 \\
\hline
\end{tabular}

Results are presented as adjusted odds ratios and $95 \%$ confidence intervals using multivariable logistic regression analysis.

SLIQ, Simple Lifestyle Indicator Questionnaire; EGF, epidermal growth factor; HOMA, homeostatic model assessment; VEGF, vascular endothelial growth factor.

6.0 to 10.8$), p=0.06)$, and no significant change in the proportion with harmful use ( $50 \%$ vs $40 \%, \mathrm{p}=0.37)$.

\section{DISCUSSION}

This study is the first to comprehensively assess the effects of short-term abstinence from alcohol in a population of 'healthy' individuals, who are representative of the $25 \%$ of the wider population who drink alcohol above national guidelines. The key findings of this study are improvements in insulin resistance, BP, body weight and a decrease in circulating concentrations of cancer-related growth factors following a month of abstinence from alcohol.

The strengths of this study are the prospective study design, the recruitment of a control group and the thorough characterisation of the participant's biological and lifestyle data. A weakness is the lack of randomisation of groups, although for ethical reasons the allocation of individuals to a predefined alcohol consumption regimen was inappropriate. A further weakness relates to the study cohort, who were recruited through staff at university teaching hospitals and a science magazine, and thus probably had higher educational attainment and health-related motivation than the average population. A further confounder is the possibility of lifestyle change in the abstinence group, alongside abstinence from alcohol. We have tried to minimise the impact of these using the SLIQ questionnaire, a self-reported measure of lifestyle factors contributing to metabolic risk with good retest reliability. ${ }^{9}$ As such, changes in HOMA score, weight and BP were independent of changes in lifestyle as measured by the SLIQ score. Nevertheless, it remains possible that the questionnaire scoring for diet, exercise and cigarette smoking has inadequate sensitivity for all lifestyle changes within this cohort.

The primary endpoint of insulin resistance, measured by HOMA score, showed a marked decrease ( 25\%) following the cessation of alcohol consumption. Some previous epidemiological data have supported a protective effect of low-dose alcohol use on the risk of type 2 diabetes, ${ }^{12}$ although more recent work suggests this may be due to incomplete adjustment for 'sick quitters', ${ }^{13}$ and prospective alcohol intervention studies have provided mixed results. ${ }^{15}$ Our data support a positive association of moderate-heavy alcohol use with an increased risk of type 2 diabetes. Moreover, the observed effects of abstinence on HOMA score noted in this study are too dramatic to be accounted for by weight loss alone, and no specific association was found between change in HOMA score and weight. To our knowledge, this is the first paper to prospectively demonstrate a link between excess alcohol consumption and insulin resistance.

A major novel finding of this study is the rapid decrease in serum VEGF and EGF with short-term abstinence from alcohol, which was seen in $90 \%$ of subjects in the abstinence group. Importantly, these changes were not seen in the control group with continued alcohol consumption. Alcohol is causally related to the development of several cancers, including the digestive tract, nasopharynx, liver and breast, and is classified as a class I carcinogen. ${ }^{46}$ The increased risk caused by alcohol persists even at low levels of consumption. The mechanism of mutagenesis is thought to relate to direct effects of the alcohol metabolite, acetaldehyde. ${ }^{4}$ However, in this study, we chose to study VEGF and EGF, since they are key molecules in the multistep progression of cancer, are both highly expressed in the solid tumours listed above and are common therapeutic targets for these tumours. ${ }^{17}$ VEGF plays a key role in tumour progression through angiogenic pathways, and VEGF expression is driven by oncogene expression (eg, Ras, src, HER2, EGFR). ${ }^{18}$ EGF signalling contributes to oncogenesis by directly promoting cell proliferation, ${ }^{19}$ and expression levels are correlated with progressive tumour growth and metastasis. ${ }^{20-22}$

Mechanistically, rodent models have demonstrated that alcohol exposure directly promotes the progression of several cancers, including breast cancer. Lu et al have shown, in a mouse model of breast cancer, that alcohol directly induces tumour angiogenesis and accelerated tumour growth through a VEGF-dependent mechanism. ${ }^{23}$ Similar evidence for an alcohol-VEGF pathway exists in mouse models of colon cancer and melanoma. ${ }^{24} 25$ The EGF pathway has also been implicated in alcohol-related breast cancer. ${ }^{26-28}$ The baseline levels of VEGF and EGF reported in this study are lower than reported in other studies exploring associations of circulating VEGF/EGF 
levels with the occurrence of solid tumours. ${ }^{29-31}$ These differences are explained by the method of sample collection. The collection of blood into EDTA tubes, as in this study, leads to reduced contribution of platelet-derived VEGF and EGF, and thus lower plasma concentrations. ${ }^{3233}$

Here, we demonstrate for the first time in humans an association of abstinence from alcohol with a marked reduction in circulating concentrations of VEGF and EGF, which suggests that alcohol consumption per se increases the concentrations of these growth factors. There is strong evidence that these growth factors play an important role in oncogenesis. However, it would be wrong to speculate further on this observation without longitudinal study in subjects who continue moderate alcohol consumption.

These data also show the dynamic effect of regular alcohol consumption on BP, an effect that is maintained in healthy individuals with no history of hypertension requiring medication. An effect of alcohol on $\mathrm{BP}$ has long been recognised, with consumption greater than two daily doses considered to be one of the most common reversible causes of hypertension. ${ }^{34}$

Collectively, the above findings have implications for the risk of synergistic liver injury among individuals with risk factors for alcohol-related liver disease (ALD) and fatty liver disease. Previous studies have emphasised an association between these pathways of liver injury, since serum ALT among moderate drinkers is elevated to a greater extent in those with higher body mass index (BMI), and ALD and fatty liver are pathologically similar. Two prospective cohort studies from Scotland have demonstrated an increased risk of liver disease with alcohol use and elevated BMI. ${ }^{35}$ More recently, a large prospective study of over 100000 women in the UK confirmed a synergistic association between alcohol and high BMI and risk of chronic liver disease. ${ }^{36}$ Since alcohol use and insulin resistance are both directly implicated in the development of steatohepatitis, the results of this study provide further support for this common causal pathway. Further, changes in the gut microbiome have also been implicated in the pathogenesis of steatohepatitis and obesity, ${ }^{37}$ and therefore changes in gut microbe populations following abstinence from alcohol are a further possible explanation for the biological changes observed in this study. These hypotheses merit further attention in subsequent mechanistic studies.

A frequent criticism of public health strategies of shortterm abstinence (eg, Dry January) has been the lack of evidence of health benefits, or even negative effects on longer-term alcohol consumption.

Although this study has demonstrated health benefits from short-term abstinence, a possible misrepresentation of these results is the concept that a 'detox' period is all that is required to 'refresh' the liver or achieve other health gains. This is clearly untrue, since the durability of the observed biological effects remains to be established. The data presented here provide supportive mechanistic evidence for the recent changes in alcohol guidance due to cancer risk, and the synergistic relationship between alcohol and metabolic syndrome. Further attention should be directed to determining the durability of these biological effects of abstinence, and conveying these complex public health messages to the public.

\section{Author affiliations}

${ }^{1}$ Division of Medicine, UCL Institute for Liver and Digestive Health, London, UK

${ }^{2}$ Camden and Islington Public Health, London, UK

${ }^{3}$ Department of Clinical Biochemistry, Royal Free London NHS Foundation Trust, London, London, UK

${ }^{4}$ UCL Tissue Access for Patient Benefit, London, UK

${ }^{5}$ Department of Anesthesiology, McGovern Medical School, University of Texas Health Science Center at Houston, Houston, Texas, USA

${ }^{6}$ Department of Liver Transplantation, HPB and Hepatology, Royal Free London NHS Foundation Trust, London, UK

${ }^{7}$ Department of Clinical Biochemistry, King's College Hospital NHS Foundation Trust London, UK

${ }^{8}$ Department of Clinical Biochemistry, Royal Free London NHS Foundation Trust London, UK

Acknowledgements Administrative support for the study was provided by Patricia Langley.

Contributors GM contributed to the study design, participated in data collection, wrote the analytical plan and drafted and revised the paper. He is the guarantor. SM participated in the study design, participated in data collection and drafted and revised the paper. AC and TK-B analysed the data, and drafted and revised the paper. CS participated in study design and revision of the paper. MR, SA-K, AJ, CC, $\mathrm{JM}, \mathrm{AG}$ and TH participated in data collection and revision of the paper. CJ, RS, DN and RJ contributed to the study design and revision of the paper. KM supervised the study, contributed to the study design, participated in data collection and drafted and revised the paper.

Funding This work was funded by the Royal Free Charity, Camden and Islington Public Health and the Royal Free London NHS Foundation Trust.

Competing interests None declared.

Patient consent Not required.

Ethics approval NRES Committee North West (14/NW/1510).

Provenance and peer review Not commissioned; externally peer reviewed.

Data sharing statement All raw data are available on request from the corresponding author.

Open Access This is an Open Access article distributed in accordance with the Creative Commons Attribution Non Commercial (CC BY-NC 4.0) license, which permits others to distribute, remix, adapt, build upon this work non-commercially, and license their derivative works on different terms, provided the original work is properly cited and the use is non-commercial. See: http://creativecommons.org/ licenses/by-nc/4.0/

(C) Article author(s) (or their employer(s) unless otherwise stated in the text of the article) 2018. All rights reserved. No commercial use is permitted unless otherwise expressly granted.

\section{REFERENCES}

1. GBD 2016 Risk Factors Collaborators. Global, regional, and national comparative risk assessment of 84 behavioural, environmental and occupational, and metabolic risks or clusters of risks, 1990-2016: systematic analysis for the Global Burden of Disease Study 2016. Lancet 2017:390:1345-422.

2. Rehm J, Samokhvalov AV, Shield KD. Global burden of alcoholic liver diseases. J Hepatol 2013;59:160-8.

3. Williams R, Aspinall R, Bellis M, et al. Addressing liver disease in the UK: a blueprint for attaining excellence in health care and reducing premature mortality from lifestyle issues of excess consumption of alcohol, obesity, and viral hepatitis. Lancet 2014;384:1953-97.

4. LoConte NK, Brewster AM, Kaur JS, et al. Alcohol and cancer: a statement of the american society of clinical oncology. J Clin Oncol 2018;36:83-93. 
5. Becker U, Deis A, Sørensen TI, et al. Prediction of risk of liver disease by alcohol intake, sex, and age: a prospective population study. Hepatology 1996;23:1025-9.

6. Dunn W, Sanyal AJ, Brunt EM, et al. Modest alcohol consumption is associated with decreased prevalence of steatohepatitis in patients with non-alcoholic fatty liver disease (NAFLD). $J$ Hepatol 2012;57:384-91.

7. Ekstedt M, Franzén LE, Holmqvist M, et al. Alcohol consumption is associated with progression of hepatic fibrosis in non-alcoholic fatty liver disease. Scand J Gastroenterol 2009;44:366-74.

8. Department of Health. UK chief medical officers' alcohol guidelines review: summary of the proposed new guidelines. https://www. gov.uk/government/uploads/system/uploads/attachment_data/file/ 489795/summary

9. Godwin M, Streight S, Dyachuk E, et al. Testing the simple lifestyle indicator questionnaire: initial psychometric study. Can Fam Physician 2008;54:76-7.

10. Sobell LCS MB. Timeline follow-back: a technique for assessing self-reported ethanol consumption. Measuring alcohol consumption: psychosocial and biological methods. Totowa: Humana Press, 1992.

11. Matthews DR, Hosker JP, Rudenski AS, et al. Homeostasis model assessment: insulin resistance and beta-cell function from fasting plasma glucose and insulin concentrations in man. Diabetologia 1985;28:412-9.

12. Baliunas DO, Taylor BJ, Irving $\mathrm{H}$, et al. Alcohol as a risk factor for type 2 diabetes: a systematic review and meta-analysis. Diabetes Care 2009;32:2123-32.

13. Knott C, Bell S, Britton A. Alcohol Consumption and the risk of type 2 diabetes: a systematic review and dose-response meta-analysis of more than 1.9 million individuals from 38 observational studies. Diabetes Care 2015;38:1804-12.

14. Schrieks IC, Heil AL, Hendriks HF, et al. The effect of alcohol consumption on insulin sensitivity and glycemic status: a systematic review and meta-analysis of intervention studies. Diabetes Care 2015;38:723-32.

15. Gepner Y, Golan R, Harman-Boehm I, et al. Effects of initiating moderate alcohol intake on cardiometabolic risk in adults with type 2 diabetes: a 2-year randomized, controlled trial. Ann Intern Med 2015;163:569-79.

16. Committee on Carcinogenicity of Chemicals in Food. Statement on consumption of alcoholic beverages and risk of cancer. $2015 \mathrm{https}: / /$ wwwgovuk/government/groups/committee-on-carcinogenicity-ofchemicals- in-food-consumer-products-and-the-environment-coc

17. Tabernero J. The role of VEGF and EGFR inhibition: implications for combining anti-VEGF and anti-EGFR agents. Mol Cancer Res 2007;5:203-20.

18. Ferrara N, Gerber HP, LeCouter J. The biology of VEGF and its receptors. Nat Med 2003;9:669-76.

19. Arteaga CL. The epidermal growth factor receptor: from mutant oncogene in nonhuman cancers to therapeutic target in human neoplasia. J Clin Oncol 2001;19(Suppl):32S-40.

20. De Jong KP, Stellema R, Karrenbeld A, et al. Clinical relevance of transforming growth factor alpha, epidermal growth factor receptor, p53, and Ki67 in colorectal liver metastases and corresponding primary tumors. Hepatology 1998;28:971-9.
21. Lo HW, Hung MC. Nuclear EGFR signalling network in cancers: linking EGFR pathway to cell cycle progression, nitric oxide pathway and patient survival. Br J Cancer 2006;94:184-8.

22. Bracher A, Cardona AS, Tauber S, et al. Epidermal growth factor facilitates melanoma lymph node metastasis by influencing tumor lymphangiogenesis. J Invest Dermatol 2013;133:230-8.

23. Lu Y, Ni F, Xu M, et al. Alcohol promotes mammary tumor growth through activation of VEGF-dependent tumor angiogenesis. Oncol Lett 2014;8:673-8.

24. Tan W, Bailey AP, Shparago M, et al. Chronic alcohol consumption stimulates VEGF expression, tumor angiogenesis and progression of melanoma in mice. Cancer Biol Ther 2007;6:1222-8.

25. Gu JW, Bailey AP, Sartin A, et al. Ethanol stimulates tumor progression and expression of vascular endothelial growth factor in chick embryos. Cancer 2005;103:422-31.

26. Ciarloni L, Mallepell S, Brisken C. Amphiregulin is an essential mediator of estrogen receptor alpha function in mammary gland development. Proc Natl Acad Sci U S A 2007;104:5455-60.

27. Willmarth NE, Baillo A, Dziubinski ML, et al. Altered EGFR localization and degradation in human breast cancer cells with an amphiregulin/ EGFR autocrine loop. Cell Signal 2009;21:212-9.

28. Meng Q, Gao B, Goldberg ID, et al. Stimulation of cell invasion and migration by alcohol in breast cancer cells. Biochem Biophys Res Commun 2000;273:448-53.

29. Burstein HJ, Chen YH, Parker LM, et al. VEGF as a marker for outcome among advanced breast cancer patients receiving antiVEGF therapy with bevacizumab and vinorelbine chemotherapy. Clin Cancer Res 2008;14:7871-7.

30. Meggiato T, Plebani M, Basso D, et al. Serum growth factors in patients with pancreatic cancer. Tumour Biol 1999;20:65-71.

31. Yoon SS, Kim SH, Gonen M, et al. Profile of plasma angiogenic factors before and after hepatectomy for colorectal cancer liver metastases. Ann Surg Oncol 2006;13:353-62.

32. Webb NJ, Bottomley MJ, Watson CJ, et al. Vascular endothelial growth factor (VEGF) is released from platelets during blood clotting: implications for measurement of circulating VEGF levels in clinical disease. Clin Sci 1998;94:395-404.

33. Yucel A, Karakus R, Cemalettin A. Effect of blood collection tube types on the measurement of human epidermal growth factor. $J$ Immunoassay Immunochem 2007;28:47-60.

34. Beilin LJ, Puddey IB. Alcohol and hypertension: an update. Hypertension 2006;47:1035-8.

35. Hart CL, Morrison DS, Batty GD, et al. Effect of body mass index and alcohol consumption on liver disease: analysis of data from two prospective cohort studies. BMJ 2010;340:c1240.

36. Trembling PM, Apostolidou S, Gentry-Maharaj A, et al. Risk of chronic liver disease in post-menopausal women due to body mass index, alcohol and their interaction: a prospective nested cohort study within the United Kingdom Collaborative Trial of Ovarian Cancer Screening (UKCTOCS). BMC Public Health 2017;17:603.

37. Henao-Mejia J, Elinav E, Jin C, et al. Inflammasome-mediated dysbiosis regulates progression of NAFLD and obesity. Nature 2012;482:179-85. 